\title{
LA GARPE DE QUALITÉ
}

\author{
Par M. DE IDROUIN DE BOUVILLE
}

Inspecteur principal de's Eaux et Forets en retraite 1 .

La Carpe de qualité n'est pas la Carpe noble. Actuellement, on n'attache plus grande importance, en ichthyotechnie, à cette question de l'ascendance, de la purcté du sang, sur laquelle on s'est longtemps hypnotisé. Plus encore aujourd hui qu'il y a quatre ans (2) nous avons la conviction que la Carpe d'élite doit ses vertus, non à sa généalogie, mais à la science et la conscience de son éleveur.

Toutefois cette Carpe d'élite - autrement dit à croissance rapide - n'est pas forcément une Carpe de qualité. Ceci parce que la plupart des éleveurs se préoccupent exclusivement de la quantité des poissons à extraire de leurs étangr. Aujourd'hui, par travail du sol, épandage d'amendements, et surtout par nourrissage de sujets maintenus en bonne forme par une sélection attentive, ou issus de croisements judicicux, on arrive à des rendements colossaux atteignant jusquà 600 kilogrammes à l'hectare (3). Mais il scmble qu'on parvienne, du mème coup, à une exploitation antiéconomique.

On commence à s'apercevoir en Allemagne, pays dont le marché règrle plus ou moins celui des autres, qu'on a trop disposé de la clientèlc. Dans l'étal de dépression économique présent, elle ne suit plus. Les cours du poisson d'étang fléchissent au-dessous du prix de revient calculé par le producteur, car il dépasse la valeur d'appréciation par le consommateur.

Des voix autorisées, nolamment celle de VoGrL (4), s’élèvent depuis quelque temps, outre khin, pour rappeler que la Carpe est un aliment relativement coûteux.

Déjà, en 1888 , le maitre pisciculteur Susta (j) faisait connaître que, pour une Carpe de 3.ō̃ grammes, les parties comestibles ne sélevaicnt qu’à r.500 grammes, soit moité. En 1899 , Lsmaxx (6) a indiqué pour des Galiciennes de 938 à r .o 8 2 grammes, une proportion de chair variant

(1) Rapport présente it la "Journén de la Carpe " a tyon, le g Novembre ig 3 ".

(2) Bullelin franģais de Piscicullure: - Novembre $9_{28}^{8}, \mathrm{n}^{\text {9 }} 5, \mathrm{p}$. 105.

(3) E. WalTER. - Die iersuche $193 \mathrm{r}$ in der bagerischen teichwirlschafllichen lersuchsanstalf Wiclenbach, - Fischerei Zeitung, Neudamm ; - $n^{\circ} 42 ; 16$ Octobre ig32, p. 498 .

(4) Korrespondenzblall für Teichwirlschaft, Gross-Tzschacksdorf ; - $\mathrm{I}_{9} 32$, passim.

(5) Die Ernïhrung der Karpfen und seiner Teichgenossen ; - Herrcke et Lebeling, Stettin, 1888 .

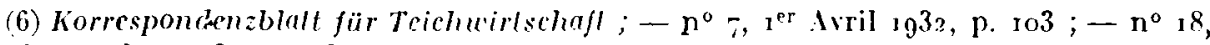
15 Septembre rg32, p. $2 S$ o. 
de 45,30 à $46,60 \%$. Kövig (I), en igr4, pour une grosse Carpe de 6.56o grammes, a trouvé $43,60 \%$ Enfin, en i 93 I, Metzyen et Rö̈ller (2), opérant plus minutieusement que leurs prédécesscurs, sont arrivés, pour un poisson du Schleswig-Holstein, à séparer 6o, 13\% de parties comestibles.

Prenons ce demier cas, le plus favorable. Lu prix de détail, plutòt bas, de 2 RM, 40 pratiqué dernièrement à Berlin, le poisson, qui pesait I.803 grammes, aurait été payé 4 R I1, 35. Pour ce prix on a r.os'z grammes de chair, y compris les arètes fines, le foie et les ceufs, dont la valeur ressort ainsi à / RM. le kilo. C'est le double, exactement, de ce qu eussent coûté, sans os, les meilleurs morćcaux de bơuf ou de pore, à la même époque, sur la même place.

Pour que semblable écart soit acecptable, il est essentiel que la Carpe soit avantageuse, plaisante, appétissante ; qu'elle flatte l'ceil et fasse venir l'eau à la bouche.

De la sorte se dégage la notion de la Carpe de qualité. Carpe délite, évidemment, mais non pousséc, forcéc, avec l'unique souci du poids fourni en un an par l'unité de surface. C'est celle qui donne, non le rendement record au producteur, mais la salisfaction optima au consommateur. Dans ces conditions, elle obtient le prix fort.

$$
\because * *
$$

Quelles seront les caractérisliques de ces Carpes recherchées des gourmets, poissons de luxe qu'on ne marchande guère?

Leur poids ; leur format; peut-îlre leur lirrée ; pilr-rlessus lout : leur saveur.

$$
\text { ***; }
$$

On doit, tout d'abord, poser en principe que la Carpe de qualité est unw Carpe lourde (Schwerliarpfen), soit un poisson de ? kilos an moins et, préférablement, de 3 kilos. C'est celui qui, voici un demi-siècle, fonda la réputation de Susta, directeur, en Bohème, d'un domaine piscicole comprenant environ ro.0oo hectares d'étangs. Celte sorte s'est raréfiée devant la submersion du marché allemand par les Carpes légères (leichte Karpfen) de $2 \overline{0}$ à r.ooo grammes dont, roici une quarantaine d'années, Bunda s'est fait le propagandiste, en vintant leur production plus facile et plus rapide. Mais les sujets de poids font toujours prime.

Prenons la répartition des Carpes en quatre catégories basécs sur le nombre de têtes aux 5o kilos, telle qu elle est adoptée à Berlin. Voici comment, d'après Vogel (3), s'établissent les prix respectifs pour des cours moyens de : - I60 RM. aux ıoo kilos pour poissons livrés gare départ par le producteur - et et 2,40 RII. all kilo pour vente au consommateur :

(1) Chemie der menschlichen Wïhrtugs-und Genussmitleln ; - Springer, Berlin, I9I/.

(2) Allgemeine Fischerei Zeilung, Augsbourg ; $-\mathrm{n}^{0} \mathbf{1}, \mathrm{r}^{\text {er }} \mathrm{Juin} \mathbf{1} 3 \mathrm{r}$.

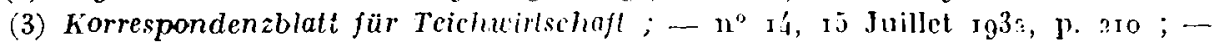
$11^{\circ} 17,1^{\mathrm{er}}$ septembre ig32, p. 260. 


\begin{tabular}{|c|c|c|}
\hline CATÉGORLS & $\begin{array}{l}\text { PRIX DACIIIT } \\
100 \mathrm{ligs}\end{array}$ & $\begin{array}{l}\text { PRIX IUE VENTE } \\
\text { Kilo }\end{array}$ \\
\hline 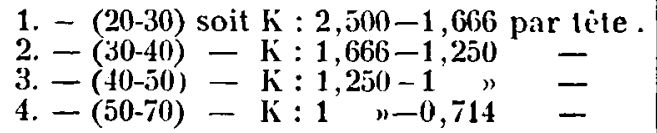 & 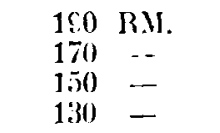 & $\begin{array}{lll}2 & 13 \mathrm{M} & 70 \\
2 & - & 50 \\
2 & - & 30 \\
2 & - & 10\end{array}$ \\
\hline
\end{tabular}

En Hongrie, les moyennes mensuclles du marché de liudapest ont varié comme suit au cours du premier semestre da l'an dernier :

Carpes grosses (Plus de 2 k.).... 1, io-? "Pengroës

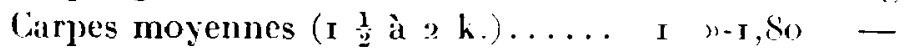

Carpes petites $\left(\frac{1}{2}-\mathbf{r}\right.$ k.)......... 0,85-1, 10 -

Enfin, au pays des Carpes monstres, on Roumanie, voici, foujours pour la période de Janvier-Juillet $x_{9} 32$, les résullats oblenus aux ventes aus enchères par les bureaux de l'Ftat :

\begin{tabular}{|c|c|c|c|}
\hline \multirow{2}{*}{ lolls } & \multicolumn{2}{|c|}{ MOVENTIS MENSCELLIFS } & \multirow{2}{*}{$\begin{array}{l}\text { MONENAL } \\
\text { SEMISSTRIEILE }\end{array}$} \\
\hline & Plus bas & Plus haut & \\
\hline $\begin{array}{l}\text { Plus de } 4 \text { kilos......... } \\
\text { De } 2 \text { à } 4 \text { kilos. . . . . } \\
\text { Moins de } 2 \text { kilos... }\end{array}$ & $\begin{array}{l}28,66 \mathrm{lci} \\
22 " 12- \\
12 " 1-\end{array}$ & $\begin{array}{l}56,28 \mathrm{~J} \cdot \mathrm{i} \\
43,14= \\
23,57 \ldots\end{array}$ & $\begin{array}{l}41,23 \text { lei } \\
31,45- \\
18,22-\end{array}$ \\
\hline
\end{tabular}

Complétant par ces dernières donnérs celles de la statislicjue allemande, on arrive à se rendre compte que la progression du prix unitaire avec laugmentation du poids par pièce est approvimalivement la suivante :

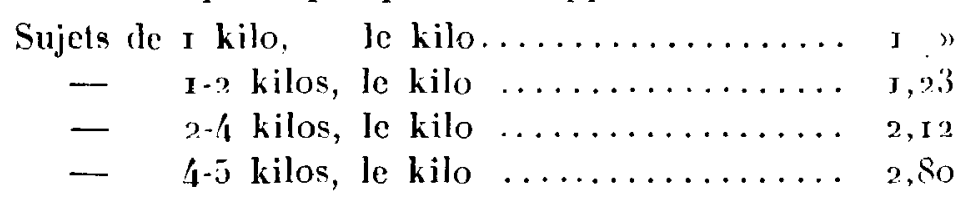

Par conséquent, la Carpe est un Poisson dont le prix an kilogramme crồt avec la taille. Il n'en va pas toujours de mime.

Ainsi, pour deux autres poissons d'élangs, voici les cours du marché de Berlin pour la période de s9?; à s $93 \%$ :

\begin{tabular}{|c|c|c|c|}
\hline \multirow{2}{*}{ ESPI:CES EYT SOHTES } & \multicolumn{2}{|c|}{ MOYENAES ANNCEILIS } & \multirow{2}{*}{$\begin{array}{c}\text { MOYENNE } \\
\text { QLINQUENNALE }\end{array}$} \\
\hline & Plus has & Plus haut & \\
\hline 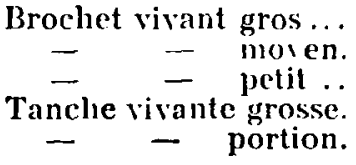 & 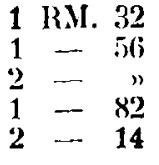 & $\begin{array}{ll}1 & \text { RM. } \\
1 & -30 \\
2 & -30 \\
2 & -70 \\
2 & -28 \\
3 & -\quad 08\end{array}$ & $\begin{array}{l}1 \text { KM. } 44 \\
1-98 \\
2-37 \\
2-03 \\
2-81\end{array}$ \\
\hline
\end{tabular}


Etant incontestable que les gourmets recherchent la Carpe lourde, consentant, pour les belles pièces, des prix rclativement élevés, c’est par son poids que se distingucra tout d'abord lil Carpe de qualité.

$$
* * *
$$

La grosseur du poisson n'est pas tout, il sied encore que soit satisfaisant l'aspect cxtérieur, on tant surtout gue révélateur de la structure intime. Or la configuration, le walbe trounent leur expression dans le format ( $\mathrm{r}$ ), qui lui-mime se définit par deux rapports de dimensions : d'abord l'indice de profil, quotient de la longueur $L_{s}(x)$ par la hauteur maxima II ; - ensuite lindice do soction, quotient de cette mome hauteur par la plus grande largerur 1.

Selon la distinction élablie en jgor par Warten (3), les Carpes d’élevage sont dites : - trapues si $\mathrm{L}, \mathrm{II}<2,60$ - normales si $?, 60<\mathrm{L}$. II $<2,80-$ enfin longues si $5.80<1 / 1 \mathrm{~L}<3$.

Par ailleurs, l veine (1) a distiugué les Carpes sveltes des Carpes râblées selon que $1 \mathrm{I} / \mathrm{l}$ est supérieur ou inféricur à $\mathrm{I}, 9^{5}$.

Sur res hases, quel sera le signialenent de la Carpe de qualite?

Pour l'elablir de fogon indisculable, it faudrait conmaître le format correspondant an pourecnlage le plus avantagenx en parties comestibles. Nous avons vu, dans re qui prérible, que daprès les constatations faites jusqu à ce jour, il variail de 43,60 à $60,13 \%$. 11 y a entre cos chiffres un écart appréciable montrant l'impertance du qabarit du poisson pour le consommateur.

On trouve, dans la lillérature, une analı̣se anatomique complète de Carpe (5).

\begin{tabular}{|c|c|c|c|}
\hline PAIRTES & EIAT FliAIS & AlRis nesesclatos & APHIS INCINIERATION \\
\hline 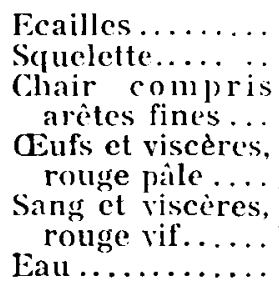 & 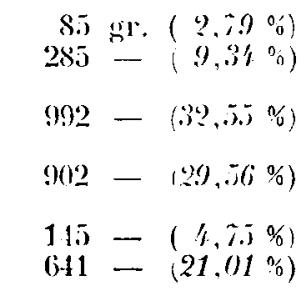 & $\begin{array}{r}43 \mathrm{gr}(6,20 \%) \\
142-(90,46 \% \\
243-(35,09 \%) \\
239-(34,43 \% \\
239-(3.89 \% \\
\%\end{array}$ & $\begin{array}{c}11,78 \mathrm{gr} .(12,39 \%) \\
54,14-(.66 .9 \% \%) \\
11,15-(11,72 \%) \\
18 \%-(18,94 \%) \\
\Rightarrow " ?\end{array}$ \\
\hline Totaux...... & 3.051 \&r. & $694 \mathrm{gr}$. & $05,07 \mathrm{gr}$. \\
\hline
\end{tabular}

(1) De Droun de lonvurs. - - Te format des Carpes, communication faite au Congrès de l'Associadion infernalionale de limnologie it liudapest, 1930 ; - voir alusi : - Dullelin frangais de Piscicullure, n० 33 . Mars $193 \mathrm{r}, \mathrm{p} .967$.

(s) Mesure prise de la pointe du musean i la nassance de la caudale.

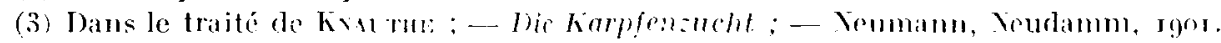

(i) Lber ingarische Edelliarpfen - horresponden-blatt für lisch:ä̈hler, Juillet ig3o

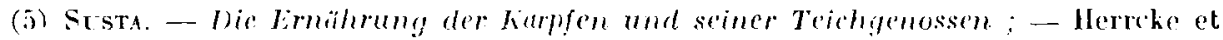
Lebeling, Stettin, y $88 S$. 
Malheurcusement, nous n avons pas le signaiement du poisson, nous savons seulement qu'il était de souche Bohémienne.

Il est cinc autres Carpes pour lespuelles, on l'a vu. divers auteurs ont donné la répartition du poids cutre les parties comestibles et les déchets, mais sans indiquer leurs dimensions (I).

Dans ces conditions, on éproune quclques embarras à dégager le format idéal de la Carpe de qualité.

Force est de s’inspirer de considérations qui n'ont pas la même autorité que des mensurations el pesées précises.

Elles amènent à négrliger l'indiec de profil et à porter spécialement lattention sur celui de scetion. Ceci parec que limportance relative des téguments et du squelette augemente apparemment avec la hauteur du corps.

Il semble qu'il faille plus d'étoffe pour habiller une Carpe svelte à dos élevé qu une Carpe rabléc à échine larréc ; on pourrait, au besoin, l'établir en étudiant la déformation d"une ellipse, courbe qui est approximativement celle limitant la section transversale dun poison. Pour un périmètre - ou tour de taille - conné, la surface circonscrite diminuc quand augmente l'écart entre les longueurs des axes.

En outre, Ies prolubérances des verlèbres : neurépines d'une part, côles pleurales de l'autre, sont plus développées chez Jo poisson à corps comprimé latéralement.

Bref, jusquà plus ample informí. il cot à croire que, plus l'indice de section est faible, plus le format est avantageux. Ajoutons avec Cxcer (o), que les poissons épais se recommandent comme élant de meilleure présentation; jls se dressent micux, et, surlout, donnent, par tronçonnagre, de plus belles tranches. Ceci n'est pas indifférent, rar la Carpe lourle est appeléc à ître débitée, soit dans les poissonneries, soit au restaurant.

Par conséquent. étant bien enlendu que le poisson de choix sera toujours un sujet d'élite caractérisé par la boses nucale, il sera d'autant mieux prisé qu’il sera plus dodu. de carrure plus massive.

D'après la documentation actucllement rassembléc, e'est entre I,60 et $\mathrm{x}, 85$ qu'oscillerait l'indice de section de la Carpe de qualité. On rencontre, comme répondant à celle formule, parmi les tỵpes bien éludiés, des Hongroises (Tala : г,6 г - Biharugra : r.85) ef certaines 1llemandes (Lausitz : т.7 $\mathbf{I}$ - Schwrarzenfold : r.S5). Il doit y avoir aussi des sortes françaises du format requis, mais, chez nous, la cyprinométric altend son protagoniste.

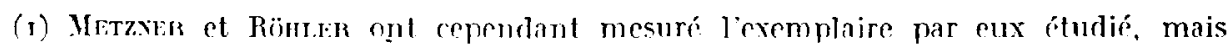
la longueur a bli prise de la pointe du musenu a lextrémiló de la queue et la hauteur en arrière de la dorsale.

(2) Magyar-Nómet nemespanly lieveztezésck : - Halàszat. Budapespt ; - no 11-12, I.j Juin $r_{932}$, p. 5 r. 
Voyons, maintenant, si la Carpe lourde et ràbléc à laquelle iront les suffrages des amalcurs doit porter une livrée spéciale.

C'est là une question débatlue en Europe centrale. Il y a les parlisans de l'Ecaille; il y a les tenants de la Miroir ou Cuir.

Les commerçants font valoir que la Carpe ordinaire, avec sa souple cuirasse, supporte mieux les transports et se conserve mieux en piscine. Tel est, entre autres, l'avis exprimé par le Syndical des poissonniers allemands (I). Il est de fait que les spécimens mi-vêtus ou nus sont exposés à lésions superficielles et que les écorchures, éraflures, desquamations les déparent fâcheusement.

Quant aux maîtres queux, ils apprécient, chez la Carpe à écailles, la consistance plus ferme de la chair, qui se tranche micux, d'où moindres déchets ; ils signalent que la peau des autres types, après cuisson, est d'un aspect déplaisant. Ainsi se résume notamment la réponse faite, lors d'une enquête, par l'Association des cuisiniers hongrois ( $\mathrm{r}$ ).

Mais si les opinions des deux corporations précédentes méritent considération, les gourmets surtout ont voix au chapitre. Or, ils ne sont pas unanimes.

Une majorité scmble pourtant so dégager en favcur de l'écaille, mais le principal grief relevé à l'encontıe de la Miroir est qu'elle est trop grasse. On le trouve nettement exprimé par des connaisseurs tels que Hacmper, Professeur à l'Ecole supérieure d'Agriculture de Vienne, et Vockr, Direc. teur de la Korrespondonsblalt fär Tcichwirtschafl, périodigue très lu cn Allemagne.

11 est possible, il y a mème des raisons de croire qu'il y a, chez les sujets à écaillure incomplète ou nulle, une certaine prédisposition à l'obésité, qui rend la chair moins appétissante ct digestible. Mais, comme on le verra par la suite, cet excès d'embonpoint n'a rien de fatal et est imputable à la suralimentalion.

Tout bien examiné, on ne voit pas de raison vraiment décisive pour disqualifier à priori une Miroir ou une Cuir. On les admeltra done à faire valoir leurs titres, quitte à discuter d'un peu plus près.

Ce qui, éminemment, fait le mérite de la Carpe de qualité, c'est sa saveur. Il est essenticl que sa dégustalion procure cette plénitude de jouissance, cette euphoric gastronomique, qu'engendrent de concert la consistance délicate de la chair et l'cxquisité de son arome.

Mais comment analyser, définir, classer les sensations gustatives ? S'il

(1) F. Zrmmer. - Carpe à écraille ou Carpe miroir ? - Athenaeum, - Budapest, 1932. 
y a l'acoustique et l'optique avec Jeurs formules algébriques, les sciences du tact, de l'odorat et du goût sont encore dans l'enfance.

Pour se faire une idéc des facteurs qui influent sur la valeur culinaire d'un aliment, on en est réduit à s'adresser à la chimic.

Par première analyse, les éléments constitutifs d'une chair quelconque se répartissent en : - cau, qu'on élimine par rlessiccation ; - matières organiques combustibles, qu'on brûle ; - substances minérales formant le résidu de l'incinération.

L'eau, insipide, ne joue, dans l'excitation des olives sensitives de certaines papilles linguales, qu un rôle d'intermédiaire, de dissolvant.

Les substances minérales, peu abondantes dans l'ensemble, se rencontrent surtout dans les parlies non comestibles du poisson. On a vu, plus haut (I), leur répartition pour une Carpe de 3.oji gr. En ajoutant aux cendres des muscles ( I gr.) celles de l'appareil génital, évaluécs ‘ $9 \mathrm{gr}$, on arrive à un total de $90 \mathrm{gr}$. environ pour les partics comestibles, soit $22 \%$ du total.

Sans doute la teneur de la chair en métallö̈des (phosphore, calcium, sodium, potassium, fluor), ou mème en métaux (fer, cuivre) doit influer quelque peu sur la saveur, mais l'action de ces corps est ignorée et paraît négligeable.

Restent les matières organiques, qui ont une prépondérance marquée. Elles se répartissent en trois groupes.

Le premier comprend les hydrocarbones ou hydrates de carbone où le carbone est associé avec l'hỵdrogène et l'oxygène, ces derniers se trouvant dans la même proportion que dans l'cau. Mais ils font défaut dans la chair de tous les poissons, tant de mer que d'eau douce.

Viennent ensuite les graisses, mélang̣es variés de palmitine $\mathrm{C}^{3} \mathrm{H}^{5}\left(\mathrm{C}^{16} \mathrm{H}^{31}\right.$ $\left.\mathrm{O}^{2}\right)^{3}$, de stéarine $\mathrm{C}^{3} \mathrm{H}^{5}\left(\mathrm{C}^{18} \mathrm{H}^{33} \mathrm{O}^{2}\right)^{3}$, et d'oléine : $\mathrm{C}^{3} \mathrm{H}^{5}\left(\mathrm{C}^{18} \mathrm{H}^{35} \mathrm{O}^{2}\right)^{3}$.

Enfin, la dernière classe comprend les matières azotées dans la composition desquelles entrent le carbone, l'hỵdrogène, l'oxygène, l'azote ef, éventuellement, le soufre et le phosphore. Ia plupart ont unc composition voisine de celle de l'albumine ou blanc d'seuf $\left(\mathrm{C}^{250} \mathrm{H}^{409} \mathrm{Az}^{67} \mathrm{O}^{81} \mathrm{~S}^{3}\right)$ et sont dites, en conséquence, albuminoïdes ou protẻiques.

Il y aurait aussi à faire mentions des vitamines, importantes au point de vue hygiénique, mais on est très mal renseignẹe à leur sujet pour ce qui concerne le poisson d'eau douce. Celles solubles dans l'cau (hydrosolubles) feraient défaut dans leur chair : seules ont été décelées les vitamines solubles dans les graisses ou liposolubles (A : antixérophtalmique ; $\mathrm{D}$ : Antirachitique). Rien ne donne à penser que ces dernières aient de l'importance au point de vue qui nous occupe.

Ces renseignements généraux permeltront d'interpréter la documen-

(r) Page 224. 
tation fournic sur la Carpe par le tableau ciraprès, qui rassemble les résultats de toutes les analyses intéressantes.

\begin{tabular}{|c|c|c|c|c|c|}
\hline ALTEURS & $\begin{array}{c}\text { Eau } \\
\%\end{array}$ & $\begin{array}{c}\text { Matières } \\
\text { a\%otées } \\
\%\end{array}$ & $\begin{array}{c}\text { Matières } \\
\text { graisses } \\
\%\end{array}$ & $\begin{array}{c}\text { Matières } \\
\text { minérales } \\
\%_{0}^{\prime}\end{array}$ & Observations \\
\hline Sista (1) $\ldots \ldots \ldots \ldots \ldots$ & 73,30 & \multicolumn{2}{|c|}{19,60} & 3,10 & Bolémicnne de $3.051 \mathrm{~g}$. \\
\hline BALLAXI) $(2 ; \ldots \ldots \ldots \ldots$ & 78,90 & 15,71 & 4,77 & 0,54 & Sans peau ni arites. \\
\hline Köxig el SilltTGLRBEK (3). & 73,34 & $20, \overline{30}$ & $6, \underline{2} 1$ & 1,22 & Poisson entier. \\
\hline KönIG (1) ........... & 76,97 & 21,68 & 1,09 & 1,33 & id. \\
\hline Kostycheff (2) ......... & 79,89 & $17, \overline{\mathrm{j}}$ & 1,42 & 1,14 & id. \\
\hline LehMaxy (1) . . . . . . & 78,85 & 17,38 & $2, \overline{5} 8$ & 1,22 & $\begin{array}{l}\text { Galicienne de } 938 \mathrm{gr} \text {. } \\
\text { non nourrie. }\end{array}$ \\
\hline Id. $\quad \ldots \ldots \cdots \cdots$ & 74,92 & 17,11 & 6,82 & 1,16 & $\begin{array}{l}\text { Galicienne de } 1.082 \mathrm{gr} \text {. } \\
\text { nourrie au lupin. }\end{array}$ \\
\hline Id. $\quad \ldots \ldots \ldots \ldots \ldots$ & 73,89 & 16,73 & 8,34 & 1,13 & $\begin{array}{l}\text { Galicionne de } 1.037 \mathrm{gr} \\
\text { nourrie de farine de } \\
\text { viande et mais. }\end{array}$ \\
\hline Id. $\quad \ldots \ldots \ldots \ldots$ & 71,60 & 16,17 & 11,13 & 1,12 & $\begin{array}{l}\text { Galicienne de } 1.057 \mathrm{gr} \\
\text { nourrje de lupin et }\end{array}$ \\
\hline ALQCiER (4) . . . . . . . . & 78,84 & 16,96 & 2,57 & 0,74 & \\
\hline SAENZ Diaz (5) . . . . . . & 71,89 & 22,56 & 0,85 & 1,70 & \\
\hline Metzxier et Röhler (1) ... & 68,34 & 13,60 & 16,11 & 2,30 & $\begin{array}{l}\text { Carpe miroir de } 1.803 \mathrm{~g} . \\
\text { du Schleswig. Hols- } \\
\text { tein, nourrie de lupin }\end{array}$ \\
\hline $\begin{array}{l}\text { (1) Loco citato. } \\
\text { (2) D'iprès Smousus : - II } \\
\text { Berlin, 1920. } \\
\text { (3) Die Bedeutung der Fisch } \\
\text { Berlin, 1909. } \\
\text { (4) Les aliments de VHomme. } \\
\text { (5) Daprès Industrias Pesqu } \\
\text { Voir aussi Bulletin franco }\end{array}$ & $\begin{array}{l}\text { zdbuch } \\
\text { rei für } \\
\text { - Paris, } \\
\text { as: } \\
\text { ide Pisc }\end{array}$ & $\begin{array}{l}\text { ler Binner } \\
\text { die Fleisch } \\
\text { igos. } \\
\text { igo, } \mathrm{n}^{\circ} \mathrm{du} \\
\text { cullure, } \mathrm{n}^{\circ}\end{array}$ & $\begin{array}{l}\text { fischerei, } \\
\text { versugung } \\
\text { 27, Septes } 19\end{array}$ & $\begin{array}{l}11, \text { p. } 1018 . \\
\text { int Deutsch } \\
\text { 10, p. } 20 . \\
\text { ibre } 1930 \text {, p. }\end{array}$ & $\begin{array}{l}\text { - Denier et Nicolas, } \\
\text { ien Reiche. - Farey, } \\
74 .\end{array}$ \\
\hline
\end{tabular}

Ces analyses, effectuées par des auteurs différents suivant des méthodes diverses, ne sont pas rigoureusement comparables, mais leur concordance, dans l'ensemble, est satisfaisante. Elles incitent à penser que la valeur gastronomique d'une Carpe tient essentiellement au rapport entre les matières azolées et les graisses.

Il faut du lard, pas trop n'en faut ;

L'excès en tout est un défaut!

Nous avons déjà vu le Professeur Hacmper reprocher à la Miroir et à la Cuir leur tendance à l'obésité ct signaler que les poissons trop gras répugnent au consommateur. Le Docteur Levext (I) a fait remarquer derniè-

(1) Le Poisson d'eau douce dans l'alimentation; la diététique. - Gazette des Hópi$\operatorname{tau} x$, Paris ; $n^{\circ} 18,2$ Mars $9^{32} 2$, p. $31 \%$. 
rement que les Carpes d'élite importées d'Europe centrale en France, grasses dans leur pays d'origine, ont été transformées par nos pisciculteurs en Poissons charnus et de saveur plus fine. Observation très exacte et qui explique la préfírence dont, en Allemagne, ces derniers sont devenus l'objet $(\mathrm{I})$.

D’après le mème auteur, comme au point de vue des sels minéraux et des matières azotées lous les poissons d'eau douce sont à peu près similaires, c'est par lcur tencur en graisse qu'ils se différencient.

Classiquement, c’est le taux de $4 \%$ qui établit unc démarcation entre les espèces dites maigres et celles qualifiées de grasses. Mais, comme on le voit au tableau précédent, pour la Carpe, le pourcentage des matières grasses varie entre 0,85 et 16, I I $\%$; elle serait donc inoerto sedis. Au vrai, dans la nature, c'est un poisson maigre, mais on peut, artificiellement, !e rendre tellement gras que les matières azotées en viennent à perdre leur habituelle prépondérancc.

Qu'il y ait, alors, cxagération, c'est ce quc montrent les analyses de Metzaner et Römler (2), qui n'ont pas opéré seulement sur l'ensemble du poisson soumis à leur expertise, mais aussi, séparément, sur les parties comestibles et les déchets. Voici les résultats obtenus :

\begin{tabular}{|c|c|c|c|c|}
\hline \multirow{2}{*}{ PARTIES } & \multirow{2}{*}{$\mathrm{EAC}$} & \multicolumn{3}{|c|}{ MATIÈRES } \\
\hline & & AZOTĹES & GRASSES & MINÉRALES \\
\hline 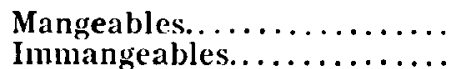 & $\begin{array}{l}71,03 \\
64,27\end{array}$ & $\begin{array}{l}14,17 \\
12,73\end{array}$ & $\begin{array}{l}14,23 \\
18,93\end{array}$ & $\begin{array}{l}1,07 \\
4,17\end{array}$ \\
\hline
\end{tabular}

On le voit, la proportion des matières grasses est appréciablement plus élevée dans les déchets. Il y a là une anomalic, micux l'indice d'un état qui, s'il n'est pas encore pathologique, le deviendra. Signalons, en passant, ce qu'a d'insalisfaisant un régime alimentaire qui alourdit surtout les parties inutilisables du poisson.

Eu égard à l'abondance des matières grasses dans un corps vivant, on distingue trois états : - la maigrreur, — l'embonpoint - et l'obésité.

Il est certain que le sujet analysé par SaEvz Diaz était étique. Mais la Galicienne non nourrie de Lenmox, dont le poids n'est inférieur que d'une centaine de grammes à celui de ses congénères suralimentées, devait ĉtre déjà en bonne condition.

Par ailleurs, la Carpe de Merziner el RönLfrest bouffie ; on peut avancer avec quasi-certitude que sa chair était flasque ct mollasse. Et, de l'avis

(1) Korrespondenzblatt für Trichwirtschaft, Gross-Tzschacksdorf; - $\mathrm{n}^{\text {o }}$ 1o et I I ;

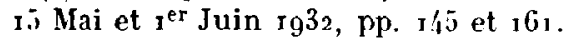

Voir aussi Bulletin français de Pisciculture, $-\mathrm{n}^{\circ} 48$, Juin $19^{32}$, p. $3-8$,

(2) Loco citato. 
de Voger (r), les sujets de I.o37 $_{7}$ et r.oj̄ grammes analysés par LemMaxi n'auraient pas été appréciés des gourmets, leur forte teneur en graisse étant écourante (Der hohe Fetlgehall... verdirbt den Appetit auf Karpfon!.

Comme conclusion, il semble que la Carpe, titrant plus de $7 \%$ de matières grasses, ne soit plus à considérer comme poisson de qualité , l'optimum, dans l'état actuel de nos connaissances, se fixerait aux alentours de $6 \%$. Avec ce taux, la chair doit avoir la consistance moellense, succulente, qui réjouit le palais des fines bouches.

Bien entendu, il n'y a pas à considérer sculement la quantité des graisses incluses dans les muscles, leur nature importe au premier chef, car c'est clle dont l'arome est surtout la manifestation.

Or, de même que l'embonpoint tient à la quotité des aliments ingérés, la qualité des matières grasses élaborées - et ceci est vrai aussi pour les matières azotées - est liée à celle de ces mêmes aliments. On ne saurait attirer trop l'attention sur ce point : la saveur dépend du régime.

On sait avec quelle facilité les tissus du poisson retiennent certains corps odorants en dissolution dans l'eau, - le pétrole par exemple - même lorsqu'ils s'y rencontrent en proportion si infinitésimale qu'ils ne sont pas décelables par l'analyse chimique (?).

Combien plus leur saveur sera-t-elle modifiéc par les substances qu'ils absorbent par les voies digestives !

Pour la Truite, quelle différence d'arome entre les sujets sauvages, se nourrissant de proies vivantes, et les élèves des salmonicultures, gavés de chairs mortes! Entre les premiers, des naturalistes gourmets font unc distinction suivant qu'ont été plutôt mises à contribution les Crevettes d'eau douce (Gammarus) ou les Larves de Névroptères (Phryganides, Perlides) (3) qui enrichissent la chair, celles-ri en matières grasses, celles-là en matières azotées. Quant aux portions des viviers, peut-on comparer celles dont la pâtée est à base de marée frâiche et celles qui reçoivent de la viande d'équarrissage ? Certains, pour tirer meilleur parti de cette dernière, n'ont-ils pas imaginé de la parfumer à l'essence de thym !

Il est certain que, pour la Carpc aussi, la chair sera d'autant plus savoureuse que la nourriture - abondante toujours - sera constituée par des animalcules vivants. C'est donc, en principe, la renonciation à l'alimentation artificielle.

Que cette dernière n'améliore pas la qualité, la preuve en est fournic par les sollicitations des poissonniers berlinois qui s'adressent aux producteurs français pour avoir de la Carpe non nourrie. C'est cette dernière

(1) Korrespondenzblatt für Teichwirtschaft, $n^{\circ} 18,15$ Seplembre 1932, p. 280 .

(2) E. Axpré. - La pollution des eaux et la chair des Poissons ; - Bulletin français de Plsciculture; $\mathrm{n}^{\circ} 43$, Janvier 1932, p. 202.

(3) Lestage. - La Truite indigène et l'Arc-en-Ciel au point de vue de leur raleur wutritive ; - Pêche et Pisciculture, Bruxelles, $n^{\circ} 7,15$ Juillet $1930, p$. 150 , 
qui a, à juste titre, les faveurs des gourmels d'Outre-Rhin. Signalons-le en passant, cette préférence est à l'origine de la campagne engagée en Allemagne pour obtenir le contingentement des poíssons d'étang. Ne voyant que les rendements colossaux à l'hectare, les carpiculteurs silésiens et bavarois jettent sur lc marché, en quantités massives, des Carpes à la fois légères et obèses. Le grand public s'en accommode quand elles deviennent attrayantes par leur bon marché ; les amaicurs demandent à l'étranger ce qu'ils ne trouvent plus chez eux. Alors, on en vient à envisager des entraves au commerce extérieur pour imposer à la consom. mation le poisson national fabriqué en série.

S'abstenir de nourrissage n'implique pas régression, abandon total de la culture intensive. Non, on gardera des procédés modernes ce qu'ils ont de foncièrement bon et l'aquiculteur, comme l'agriculteur, sollicitera son fonds par des façons culturales et surtout par des amendements.

Ce que peuvent donner ces derniers, nous l'apprenons par les comptes rendus annuels de la Station bavaroise de recherches carpicoles, à Wiclenbach. Citons, par exemple, les résultats de $\mathrm{r} 93 \mathrm{r}$, tout dernièrement publiés par Waiter ( $\mathrm{I}$ ). Ils concernent des étangs d'expérience ayant reçu l'empoissonnement dit normal, savoir: par hectare:- I 20 Carpes de 2 étés, - I 40 Carpes de I été, - 6o Tanches de I été.

\begin{tabular}{|c|c|c|c|c|c|}
\hline \multirow{3}{*}{$\begin{array}{l}\text { NOMBRE } \\
\text { D'EXPÉ- } \\
\text { HIENCES }\end{array}$} & \multirow{3}{*}{$\begin{array}{l}\text { AMENDEMENTS } \\
\text { (Doss in l'Hect.) }\end{array}$} & \multicolumn{4}{|c|}{ ACCROISSEMENT } \\
\hline & & \multicolumn{3}{|c|}{ PAI TÈTE } & \multirow{2}{*}{$\begin{array}{l}\text { TOTAL } \\
\text { a l'Hect. }\end{array}$} \\
\hline & & $\begin{array}{l}\text { CARPES } \\
2 \text { etés }\end{array}$ & CARPES & $\underset{1 \text { èté }}{\text { TANChes }}$ & \\
\hline 3 & Néant (contròle).. & $458 \mathrm{gr}$. & $309 \mathrm{gr}$. & $111 \mathrm{gr}$. & $100 \mathrm{kgs}$ \\
\hline 3 & Basiphosphate : $125 \mathrm{kgs} . . .$. & $837-$ & $458-$ & $160-$ & $163-$ \\
\hline 2 & $\begin{array}{l}\text { Basiphosphate : } 125 \mathrm{kgs} \ldots \ldots \\
\text { iChlorure de potassium : } 250 \mathrm{kgs} \text {. }\end{array}$ & $925-$ & $536-$ & $144-$ & $182-$ \\
\hline 1 & Poudre d'os Aiera ": 150 kgs. & $782-$ & $458-$ & $120-$ & $145-$ \\
\hline
\end{tabular}

On a, antérieurement, enregistré des rendements encore plus satisfaisants : avec le guano on est arrivé, cn i 930 , à doubler au moins l'accroissement naturel (Naturalzurvachs).

Ceci paraît suffisant.

Ajoutons, cependant, que si, dans certains cas exceptionnels, il devenait nécessaire de donner un complément d'alimentation à la Carpe de qualité, jl faudrait intervenir très modérément avec des produits de premier choix. Ce serait une erreur de recourir, par exemple, à des grains altérés, à des farines avariées.

Par ailleurs, les gourmets n'admettent pas l'emploi du maïs ; au vrai, ils ne tolèrent que celui du lupin.

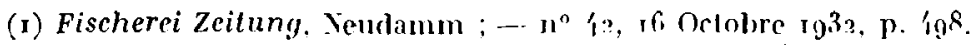


Résumons.

La Carpe de qualité est un poisson : - lourd, pesant ses 2 kilos, sinon plus, - à bosse nucale, - râblé, c'est-à-dire ayant un indice de section compris entre 1,60 et 1,85 , - présentant de l'embonpoint, sans plus, ce qui implique une teneur en matières grasses inférieure de moitié au moins à celle en matières azotées et voisine de $6 \%$, par suite non nourri, au moins dans la règle.

Parcille Carpe sera, évidemment, de production relativement onéreusc, mais trouvera preneur à bon prix.

A ce poisson de luxe des égards dont dus.

Il réclame une préparation très soigneuse, mais raffinéc dans sa simplicité, car il faut se garder d'éteindre son arome sous les épices d'une de ces sauces qui, suivant l'expression courante, font manger le poisson. Il sied, au contraire, de mettre en valeur cette saveur propre de la Carpe extra ; son accommodement devra être ce qu'est l'accompagnement d'un orchestre de choix à la voix de la prima dona. A ce sujet, les cordons bleus consulteront avec fruit le gastronome logicien qui se dissimule sous l'énigmatique formule : J. M. X. 97 ( 1 ).

Enfin, quand paraît sur la table du festin le plat bien dressé, bien paré, c'est le moment de déboucher la bouteille de derrière les fagots. Maledictus: piscis in aqua terlia ! disent plaisamment les Hongrois. Honnie soit la Carpe qui, après l'eau de l'étang et celle du court-bouillon, en connaîtrait une troisième I En France nous n'avons que l'cmbarras du choix entre les crus généreux. Mais, s'il est plus d'un moyen de réaliser l'accord parfait entre la Carpe de qualité et le vin de grande classe, il en est un, rationnel semble-t-il, qui mérite d'ètre signalé en conclusion. Est-il gourmet, mème ennemi du trône et de l'autel, qui resterait rebelle à l'harmonic de la Royale Neufbourg et du Chàteauncuf pontifical ?

\section{GONSULTATIONS TEGHNIQUES}

5) D. - Qu'est-ce que le poisson dénommé Sandre dont les oours sont donnés par le Bulletin pour certaines places étrangères? Scrait-il susceptible d'élevage dans l's étangs français? -. (P. P., a Paris).

5) R. - Le Sandre (Sander Lucioperca. L.), dit aussi Brochet-Perche, ou encore, de son appellation magyare : Fogast h ou Fogosch, est un Percide de l'Europe centrale et orientale, où il se trouve dans les lacs et les grands cours d'eau.

Cette espèce. dont la chair est très estimée, est l'objet d'un élevage en eau douce ou saumâtre en Allemagne, Hollande, Hongrie, elc. Quelques essais ont été tentés dans notre pays, mais ont été arrêtés par la difficulté de fournir, économiquement, une quantité suffisante de blanchaille à un vorace de robuste appétit.

(1) Recettes de cuisine raisonnées : la Carpe; - Poirier, Gien, 1932. 